\title{
Advantages of the Combination of Conscious Sedation Epidural Anesthesia Under Fluoroscopy Guidance in Lumbar Spine Surgery
}

This article was published in the following Dove Press journal:

Journal of Pain Research

\author{
Seung Youn Kang' \\ Osama Nezar Kashlan ${ }^{2}$ \\ Ravindra Singh ${ }^{2}$ \\ Rahul Rane $\mathbb{D}^{2}$ \\ Nitin Maruti Adsul ${ }^{3}$ \\ Sung Chan Jung' \\ Jihwan $\mathrm{Yi}^{\prime}$ \\ Hae Sun Cho' \\ Hyeun Sung Kim (D) ${ }^{2}$ \\ II-Tae Jang ${ }^{2}$ \\ Seong-Hoon $\mathrm{Oh}^{4}$ \\ 'Department of Anesthesiology, Nanoori \\ Hospital Gangnam, Seoul, Republic of \\ Korea; ${ }^{2}$ Department of Neurosurgery, \\ Nanoori Hospital Gangnam, Seoul, \\ Republic of Korea; ${ }^{3}$ Department of \\ Orthopedics, Ganga Ram Hospital, Delhi, \\ India; ${ }^{4}$ Department of Neurosurgery, \\ Nanoori Hospital Bupyeong, Incheon, \\ Republic of Korea
}

Correspondence: Hyeun Sung Kim Department of Neurosurgery, Nanoori Hospital Gangnam, \#06048 73I Eonju Street, Gangnam-Gu, Seoul, Republic of Korea

Tel +82 260039767

Fax +82 234459755

Email neurospinekim@gmail.com
Background: With the increase in life expectancy seen throughout the world, the prevalence of degenerative spinal pathology and surgery to treat it has increased. Spinal surgery under general anesthesia leads to various problems and complications, especially in patients with numerous medical comorbidities or elderly patients. For this reason, there is a need for safer anesthetic methods applicable to unhealthy, elderly patients undergoing spinal surgery.

Purpose: To report our experience with utilizing fluoroscopy-guided epidural anesthesia in conjunction with conscious sedation in spinal surgery.

Patients and Methods: We performed a retrospective review of 111 patients at our institution that received fluoroscopy-guided epidural anesthesia for lumbar surgery from February to September 2018. Patients' records were evaluated to evaluate patient demographics, American Society of Anesthesiology Physical Classification System (ASA) class, and pain numerical rating scores (NRS) preoperatively and throughout their recovery postoperatively. Intraoperative data including volume of epidural anesthetic used, extent of epidural spread, and inadvertent subdural injection was collected. Postoperative recovery time was also collected. Results: The mean age of our patients was 60 years old with a range between 31 and 83 years old. All patients experienced decreases in postoperative pain with no significant differences based on age or ASA class. There was no association between ASA class and time to recovery postoperatively. Older patients (age 70 years or greater) had a significantly longer recovery time when compared to younger patients. Recovery also was longer for patients who received higher volumes of epidural anesthesia. For every $1 \mathrm{~mL}$ increase of epidural anesthetic given, there was an increase in the extent of spread of 1.8 spinal levels. Conclusion: We demonstrate the safety and feasibility of utilizing conscious sedation in conjunction with fluoroscopy-guided epidural anesthesia in the lumbar spinal surgery.

Keywords: fluoroscopy-guided epidural anesthesia, conscious sedation, endoscopic decompressive lumbar spine surgery, capnogram monitoring

\section{Introduction}

General anesthesia is most commonly used in lumbar spine surgery due to many advantages including greater patient tolerance, the presence of a secure airway, the facilitation of spine exposure due to administration of muscle relaxants, the ability to perform a robust postoperative exam without clouding from traditional epidural anesthetic agents, and greater ease to control any transient patient hemodynamic changes intraoperatively. ${ }^{1,2}$ However, this type of anesthesia also has its inherent risks, especially in older patients. ${ }^{3-10}$ For these reasons, many Korean neurosurgeons have begun utilizing epidural anesthesia for most lumbar surgeries. 
Historically, epidural anesthesia is not preferred for lumbar surgery because of its slower onset and mixed success rate, likely due to incomplete puncturing of the ligamentum flavum by inexperienced performers ${ }^{11-15}$ and compromising intra-operative neuro-monitoring such as SSEP, MEP. Another issue with epidural anesthesia that causes relatively high failure rates is the inconsistency of the cranial extent of epidural extension. ${ }^{16-18}$ A solution to assist with overcoming the two stated challenges is the use of contrast dye intraoperatively in conjunction with fluoroscopy. Injecting dye to confirm placement and cranial extent of epidural drugs has improved the success rates of performing lumbar surgery under this modality. Moreover, when used in a dilute formation, patients can undergo major lumbar procedures and continue to have a postoperative neurologic examination to check soon after the completion of the procedure. The purpose of this study is to present our clinical experience in fluoroscopy-guided epidural anesthesia combined with conscious sedation in the treatment of patients undergoing lumbar decompressive or fusion surgery with the goal of demonstrating that epidural anesthesia is a safe and effective method that can be used in this patient population.

\section{Materials and Methods}

\section{Study Population}

We performed a retrospective review of the hospital records of all patients undergoing elective lumbar spine surgery under epidural anesthesia utilizing fluoroscopic guidance and confirmation at our institution between February and September 2018. All procedures performed in studies involving human participants were in accordance with the ethical standards of the Nanoori Hospital's Ethics Committee (NRIRB 2018-017) and the national research committee and with the 1964 Helsinki declaration and its later amendments or comparable ethical standards. Also Written informed consent was obtained from all individual participants included in the study. Expert senior surgeons and anesthesiologists utilizing fluoroscopy guidance with contrast dye image confirmation performed all the epidural anesthesia. Exclusion criteria included expected surgical time greater than $4 \mathrm{hrs,}$, anterior lumbar interbody fusion, surgeries performed with only local anesthesia, any surgery level above the T12-L1 disk space, and patients with American Society of Anesthesiologist Physical Classification System (ASA) class of IV or above. The patients underwent endoscopic lumbar decompression, microscopic discectomies and decompressions, unilateral approach to bilateral decompression and fusion, and posterior lumbar fusion including patients undergoing multilevel operations. Patient baseline characteristics were obtained via chart review including patient age, and ASA class.

\section{Anesthetic Technique and Postoperative} Course

Patients were brought into the operating room where they were placed in the prone position. Their faces were placed in a hollowed out rectangular face protection cushion. They all had 3-6 L of oxygen supply administered by nasal cannula or facemask. After injecting local anesthetic at the skin, a 20gauge Tuohy needle was introduced into the epidural space at the nearest interlaminar epidural space cranial or caudal to the operative level under fluoroscopy guidance. After the epidural space was identified with the loss-of-resistance technique and confirm the epidural placement with anteroposterior (AP) and lateral of fluoroscopy, a single injection of concoction of 5-10 mL of half $0.325 \%$ Ropivacaine with 1:200,000 epinephrine diluted in 5-10 mL of a radiocontrast dye $\left(\right.$ BONOREX $^{\circledR}$ ) was administered (Figure 1). Under fluoroscopic guidance, we infused the epidural canal until it reached the T12-L1 disk space, a level that would definitely cover the surgical field. In cases of revision surgery where there are significant epidural adhesions inhibiting the spread of dye cranially, we repeated this puncture superior to the level of epidural dye spread inhibition. Similarly if the epidural canal did not fill caudal to the operative level due to severe stenosis, a second epidural injection was performed inferior to the operative level to ensure adequate epidural dye throughout the lumbar spine. Even though many of our patients had undergone revision surgery or had severe stenosis, performing epidural anesthesia at multiple levels was a rare occurrence. If we entered the subarachnoid space, we would remove the needle and attempt our epidural injection again. However, if we inadvertently entered the subdural - but not subarachnoid space as signified by a high-density lentiform contrast stain is seen near posterior margin of the dural sac and nerve roots cannot be identified on fluoroscopy,${ }^{19}$ we would continue the anesthetic administration and note that our anesthesia was subdural rather than epidural (Figure 2).

After the epidural was given, we closely monitored patients' vital signs and comfort, especially the eyes and arms, for 5 to 10 mins. If a patient was deemed stable and comfortable, we placed a capnogram monitor on the patient's cheek or under the nose to monitor patient's respiratory status. The hollowed-out face cushion created a small pseudo-sealed 


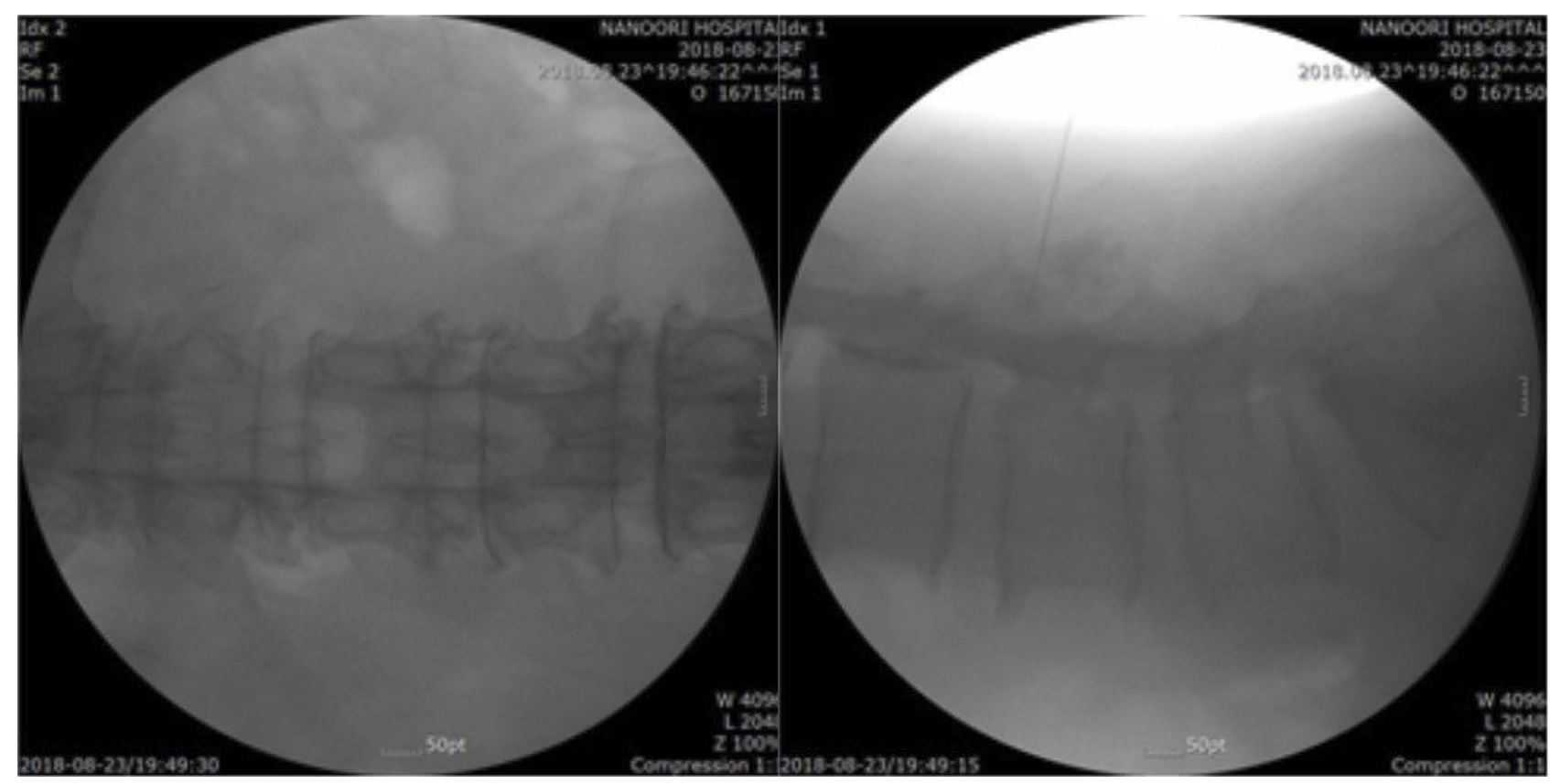

Figure I AP and Lateral of epidural solution (radiocontrast dye + local anesthetic) image by fluoroscopy.

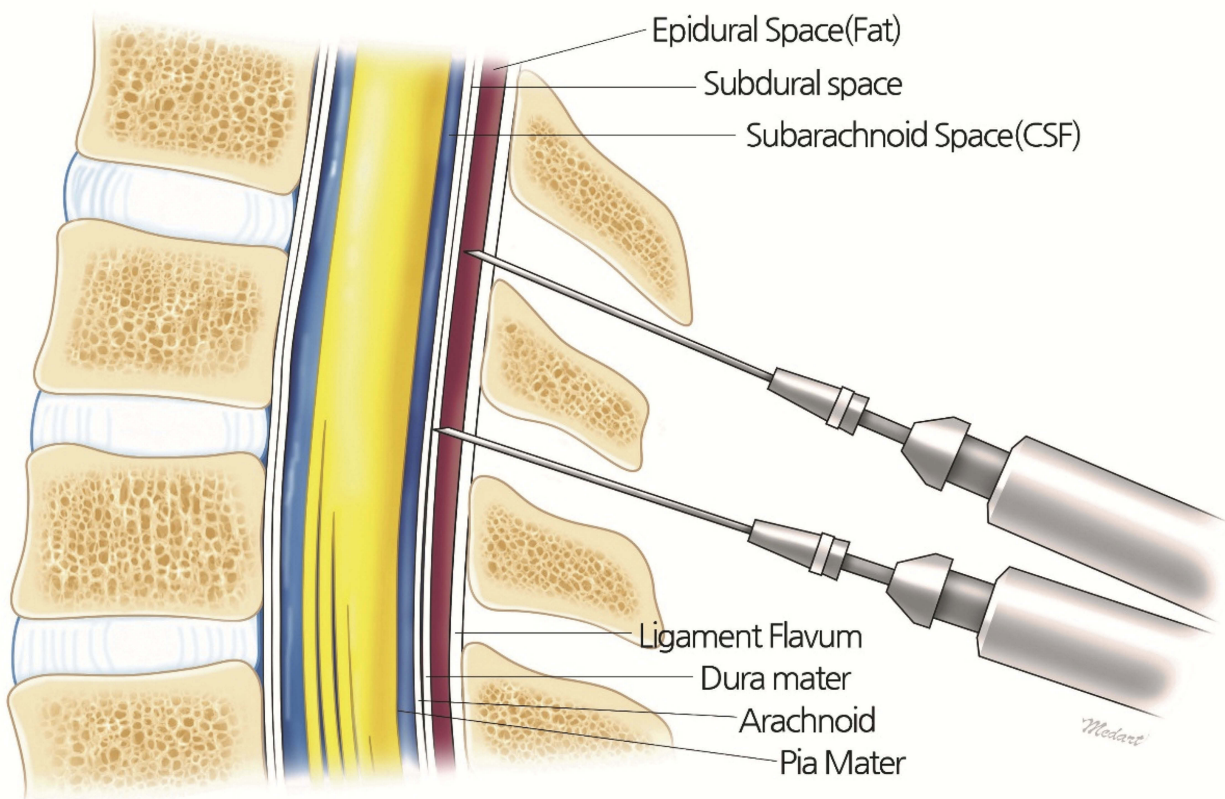

Figure 2 Anatomy of Epidural-Subdural-Subarachnoid space (from outside to inside).

chamber for the capnogram to obtain readings from (Figure 3). We then started conscious sedation by loading dose of $0.6-1$ $\mathrm{mcg} / \mathrm{kg}$ dexmedetomidine $\left(\right.$ Precedex $^{\circledR}$ ) for $10 \mathrm{mins}$ and followed by maintenance dose of $0.4-0.6 \mathrm{mcg} / \mathrm{kg}$ dexmedetomidine infusion in addition to 1 to $2 \mathrm{mg}$ of midazolam depending on patient's age, gender and adjusted body weight. Administration of additional $1 \mathrm{mg}$ doses of midazolam was continued hourly. In some patients refractory to the dexmedetomidine and midazolam, we started $60-100 \mathrm{mcg} / \mathrm{hr}$ remifentanil infusion also. Further dosing of these medications was adjusted by checking the patient's respiratory status and degree of sedation.

Postoperatively, patients were transferred to the PACU where we checked their basic vital signs, neurologic examination, and pain levels including numerical rating scores (NRS). If patients were not fully awake after 30 mins of 


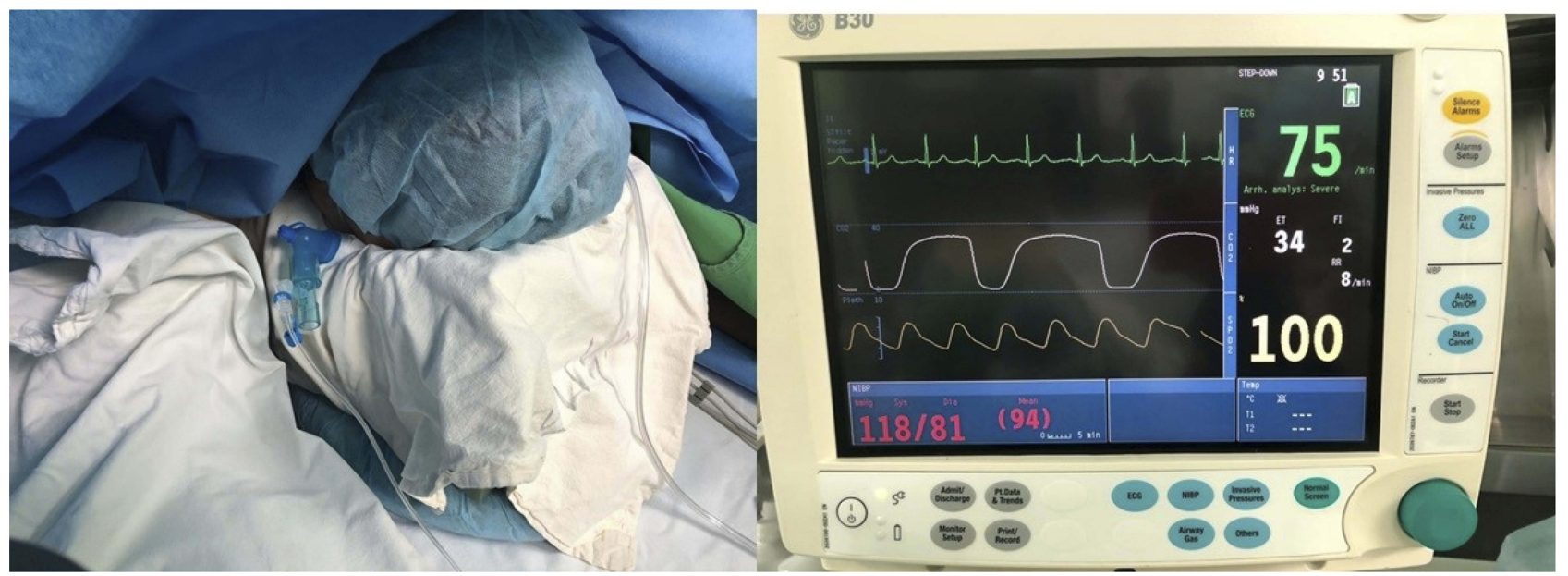

Figure 3 Capnogram monitoring during conscious sedation.

observation, we administered $1 / 2$ to 1 ample of intravenous flumazenil slowly to antagonize residual midazolam. Patients were then transferred to their ward after they were awake, able to follow verbal orders, and their motor function is able to be evaluated accurately. This time was defined as recovery time in this study. On the ward, NRS scores, vital signs, and neurologic evaluations were again checked regularly. After discharge, patients were seen in clinic 1-month postoperatively and were asked about their NRS score at that time.

\section{Statistical Analysis}

In the statistical analysis, the Independent $t$ test, paired $t$ test, ANOVA and simple regression were used. $P$ values less than 0.05 were considered significant.

\section{Results}

\section{Patient Baseline Characteristics}

There were a total of 111 patients enrolled, with a mean age of 60 years and an age range from 31 to 83 years. We split patients into three age groups: $30-49,50-69$, and 70 and over. There were 22, 61 and 28 patients in the first, second, and third groups, respectively. The study population included 62 females and 49 males. Thirty-two underwent microscopic decompressive procedures including laminectomies, laminotomies and discectomies. Sixty-one underwent endoscopic decompressive procedures. Eighteen underwent open fusion procedures. Fifteen patients were ASA class 1, 83 were ASA class 2, and 14 were ASA class 3 (Table 1). Out of the 111 patients, 8 patients were administered subdural - but not subarachnoid - anesthetic. There were no adverse events related to epidural anesthesia and no inadvertent subarachnoid infusions. All patients had complete epidural coverage with dye from the distal sacrum to the T12-L1 disk space.

\section{Procedures Utilizing Epidural Anesthesia Achieve Immediate Adequate Pain Relief}

There was statistically significant improvement $(\mathrm{p}<0.001)$ in NRS immediately postoperatively, on postoperative day 1 , on the day of discharge and at the 1 month visit (Figure 4).

Table I Demographic Data for Categorical and Continuous Variables

\begin{tabular}{|l|l|l|l|}
\hline & N & Mean (SD) & Percentage (\%) \\
\hline Age (Years) & 111 & $60.4(12.8)$ & N/A \\
$30-49$ & 22 & & \\
$50-69$ & 61 & & \\
Over 70 & 28 & & \\
\hline Sex & 111 & N/A & \\
Female & 62 & & 55.9 \\
Male & 49 & & 44.1 \\
\hline Operation & 111 & N/A & \\
Open Decompression & 32 & & 28.8 \\
Endoscopic Decompression & 61 & & 55.0 \\
Open Posterior Fusion & 18 & & 16.2 \\
\hline Anesthesia Epidural & 111 & N/A & \\
Epidural & 103 & & 92.8 \\
Subdural & 8 & & 7.2 \\
\hline ASA class & 111 & N/A & \\
I & 15 & & 13.5 \\
2 & 83 & & 74.8 \\
3 & 14 & & 12.6 \\
\hline
\end{tabular}

Abbreviations: N, number; SD, standard deviation; N/A: not applicable. 


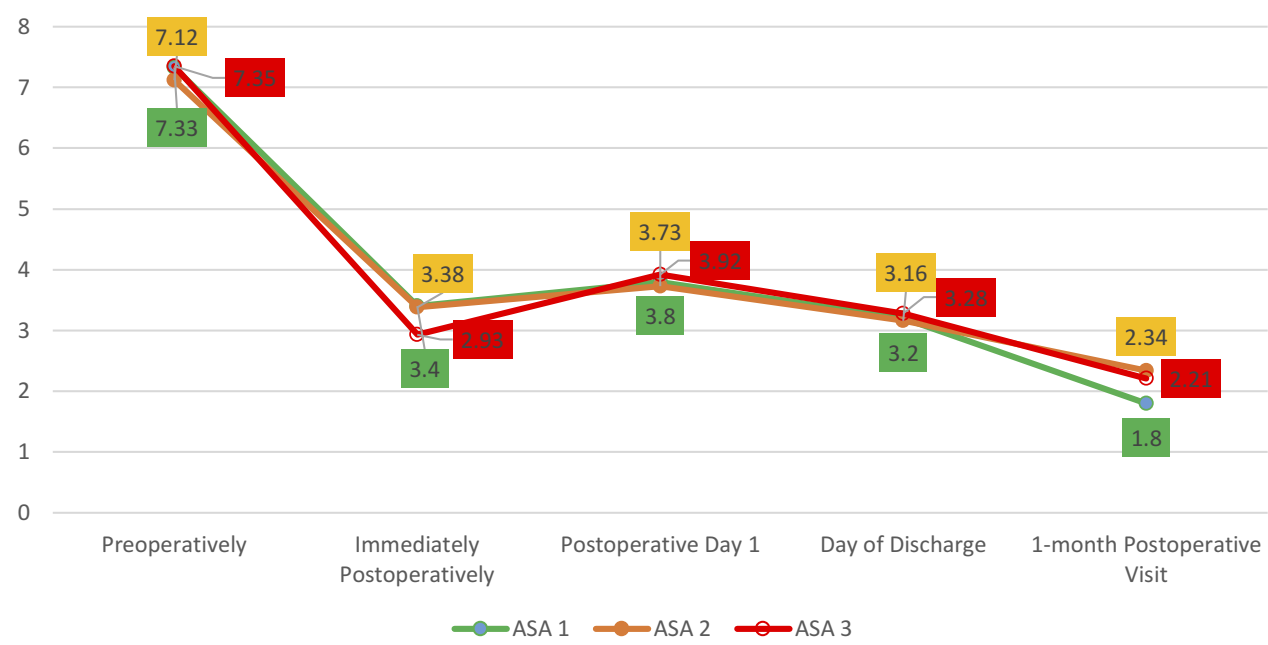

Figure 4 Improvement of NRS score with respect to ASA class.

Abbreviations: NRS, numeric rating scale; ASA, American society of anesthesiologists classification.

This effect was present in all types of surgeries including fusion surgeries, which utilizing traditional anesthetic methods typically have high pain scores immediately postoperatively (Figure 4). There was no difference in pain relief at all time points between patients in the different age groups or between patients with ASA class I, II or III (Tables 2 and 3).

Table 2 NRS Mean Difference for Each ASA Class Groups

\begin{tabular}{|l|l|l|l|l|l|l|}
\hline $\begin{array}{l}\text { (A) } \\
\text { ASA }\end{array}$ & $\begin{array}{l}\text { (B) } \\
\text { ASA }\end{array}$ & $\begin{array}{l}\text { Mean } \\
\text { Difference } \\
\text { (A-B) }\end{array}$ & $\begin{array}{l}\text { Std. } \\
\text { Dev }\end{array}$ & P-value & \multicolumn{2}{|l|}{$95 \%$ CI } \\
\cline { 5 - 7 } & & & $\begin{array}{l}\text { Low } \\
\text { Limit }\end{array}$ & $\begin{array}{l}\text { High } \\
\text { Limit }\end{array}$ \\
\hline ASA I & ASA 2 & 0.76 & 0.41 & 0.157 & -0.21 & 1.74 \\
& ASA 3 & 0.39 & 0.54 & 0.754 & -0.90 & 1.68 \\
\hline ASA 2 & ASA 1 & -0.76 & 0.41 & 0.157 & -1.74 & 0.21 \\
& ASA 3 & -0.37 & 0.42 & 0.655 & -1.38 & 0.63 \\
\hline ASA 3 & ASA 1 & -0.39 & 0.54 & 0.754 & -1.68 & 0.90 \\
& ASA 2 & 0.37 & 0.42 & 0.655 & -0.63 & 1.38 \\
\hline
\end{tabular}

Abbreviations: NRS, numeric rating scale; ASA, American society of anesthesiologists classification; Std. Dev, standard deviation; C.I, confidence interval.

\section{Recovery Time Is Longer for Patients Aged 70 Years and Older}

Recovery time, defined as time for patients to be completely awake and have a baseline neurologic examination postoperatively, was found to be longer in patients in the older age group when compared to the group aged 30-49 years $(p=0.024)$. This effect was not seen in the 50-69 year age group (Figure 5). ASA class and type of surgery (open decompression, endoscopic decompression, or open fusion) did not affect recovery time (Table 4).

\section{Increasing Volumes of Epidural Anesthesia Increase Extent of Spread and Recovery Time}

As we increased the volume of solution injected, the anesthetic spread over a greater surface area of epidural space as seen on AP and lateral fluoroscopy (Figure 6). The slope of the line of best fit demonstrated that for every

Table 3 NRS Mean Difference for Age Groups

\begin{tabular}{|l|l|l|l|l|l|l|}
\hline (A) Age Group & (B) Age Group & Mean Difference & Std. Dev & P-value & \multicolumn{2}{|l|}{$95 \%$ C.I } \\
\cline { 3 - 7 } & & (A-B) & & & Low Limit & High Limit \\
\hline $30-49$ & $50-69$ & 0.17 & 0.37 & 0.890 & -0.71 & 1.04 \\
& Over 70 & 0.48 & 0.42 & 0.486 & -0.51 & 1.48 \\
\hline $50-69$ & $30-49$ & -0.17 & 0.37 & 0.890 & -1.04 & 0.71 \\
& Over 70 & 0.31 & 0.33 & 0.620 & -0.48 & 1.11 \\
\hline \multirow{2}{*}{ Over 70 } & $30-49$ & -0.48 & 0.42 & 0.486 & -1.48 & 0.51 \\
& $50-69$ & -0.31 & 0.33 & 0.620 & -1.11 & 0.48 \\
\hline
\end{tabular}

Abbreviations: NRS, numeric rating scale; ASA, American society of anesthesiologists classification; Std. Dev, standard deviation; Cl, confidence interval. 


\section{recovery time}

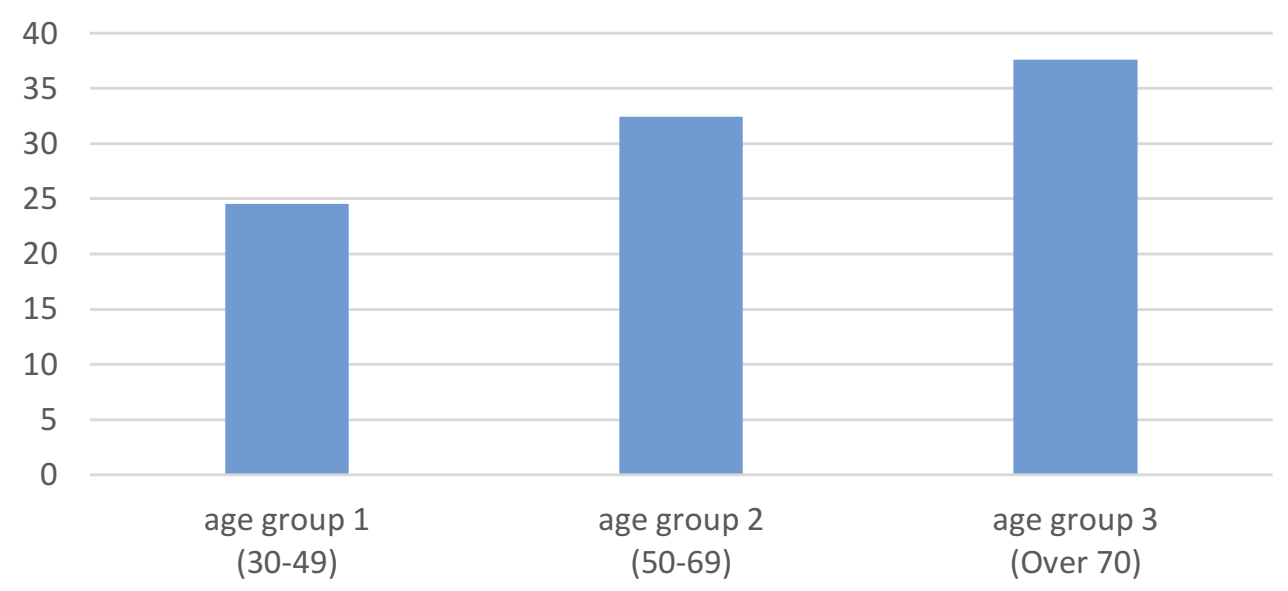

Figure 5 Recovery time (minutes) among age groups.

$1 \mathrm{~mL}$ increase of epidural anesthetic given, there was an increase in extent of spread of 1.8 spinal levels. The volume of anesthetic administered was also found to be directly related to recovery time. To the limits of the small number of patients with subdural administration, subdural anesthesia did not affect time to recovery.

\section{Discussion}

In this case series, we show that epidural anesthesia in conjunction with conscious sedation is a safe and feasible approach to treating patients of all ages and an ASA class III or less undergoing lumbar spinal surgery less than $4 \mathrm{hrs}$ in procedural length. Procedures that are safe to be performed under this anesthesia approach include open decompression operations, endoscopic decompressions, and open fusions. With epidural anesthesia in 111 patients, we had no adverse events and all patients had complete coverage with radiolucent dye from the sacrum to the T12-

Table 4 Recovery Mean Difference for ASA Class Groups

\begin{tabular}{|l|l|l|l|l|l|l|}
\hline (A) & (B) & Mean & Std. & P-value & \multicolumn{2}{|l|}{$95 \%$ Cl } \\
\cline { 5 - 7 } & ASA & $\begin{array}{l}\text { Difference } \\
\text { (A-B) }\end{array}$ & Dev & & $\begin{array}{l}\text { Low } \\
\text { Limit }\end{array}$ & $\begin{array}{l}\text { High } \\
\text { Limit }\end{array}$ \\
\hline ASA I & ASA 2 & -7.80 & 4.98 & 0.265 & -19.64 & 4.04 \\
& ASA 3 & -8.95 & 6.60 & 0.368 & -24.64 & 6.73 \\
\hline ASA 2 & ASA 1 & 7.80 & 4.98 & 0.265 & -4.04 & 19.64 \\
& ASA 3 & -1.15 & 5.13 & 0.973 & -13.35 & 11.04 \\
\hline ASA 3 & ASA 1 & 8.95 & 6.60 & 0.368 & -6.73 & 24.64 \\
& ASA 2 & 1.15 & 5.13 & 0.973 & -11.04 & 13.35 \\
\hline
\end{tabular}

Abbreviations: ASA, American society of anesthesiologists classification.

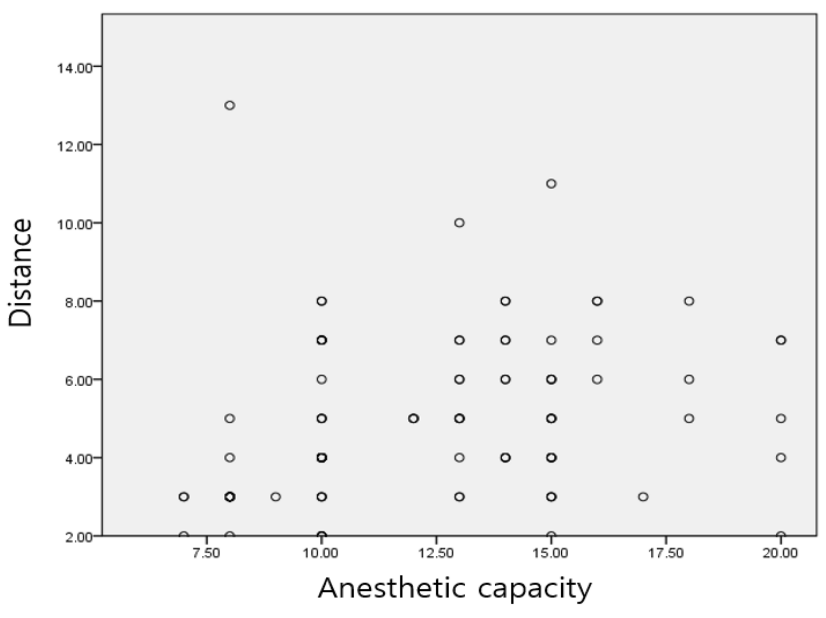

Figure 6 The volume of anesthetic injection and epidural spread by spine level.

L1 disk space cranially with mostly one or two epidural access points, even in patients with significant epidural adhesions and central stenosis. All patients had adequate pain control immediately postoperatively, and recovery times were reasonable as to give the surgeon the ability to monitor a postoperative examination soon after the end of surgery.

One of the key technical points that had a large part to do with our high success rate with no adverse events was the use of AP and lateral fluoroscopy in confirming epidural placement and spread of solution to cover the sacrum to the T12-L1 disk space cranially.

From our experience, there are 5 advantages of fluoroscopic guidance epidural anesthesia when compared to conventional blind methods. First, fluoroscopic guidance epidural anesthesia is complete and precise methods, even 
in cases of revision surgery where there are significant epidural adhesions inhibiting the spread of dye, could anesthetize full coverage of epidural space, if that happens, we repeated a second epidural injection superior or inferior to the level of epidural dye spread inhibition to ensure adequate epidural dye throughout the lumbar spine. Second, we could know the success of epidural procedure right away under this modality, which means we do not have to wait until 20 to 30 mins to confirm the perfection of epidural anesthesia without confidence. Third, once epidural anesthetic reached from the sacrum to the T12-L1 disk space, we stopped injecting more solution that made us using as small amount as 7 milliliters of epidural solution totally, which means at least 3.5 milliliters of local anesthetic was needed full coverage of the surgical field, making less chance of local toxicity complications. Fourth, with fluoroscopic view, we could adjust the anesthetic level. Once we found that the epidural anesthetic extending too cranially (high anesthetic level) by fluoroscopy, then we changed the patient's bed to the head-up position to encourage epidural anesthetic moving caudally.

Lastly, fluoroscopic guidance epidural anesthesia is a very safe and precise anesthetic method. Based on our clinical experience, in some patients, if we failed to find the epidural space by loss-of-resistance technique whose ligament flavum was very thin, and when the tip of needle was located in between subdural and subarachnoid space, inadvertent subarachnoid injection could happen, although there was no CSF aspiration. Without fluoroscopy, this could hazard the patient such as total spinal anesthesia with as much as $10 \mathrm{~mL}$ or more of local anesthetic injection if performed blindly.

In 8 patients, we did place our anesthetic in the subdural but not subarachnoid - space. This was seen on fluoroscopy as streaks of high homogeneous density, elongated and usually located at the posterior or posterolateral margins of the dural sac and does not extend to outline the exiting spinal roots laterally. ${ }^{19}$ With the limitations in size, we did not see any episodes of cardiopulmonary collapse after subdural injection, likely meaning that this effect is only seen after subarachnoid injection. Subdural anesthesia had a trend towards longer recovery times, likely due to retention of the anesthetic that would normally be washed out in the epidural space after decompression by the surgeon, but this trend was not statistically significant. However, further studies with a larger sample size would be able to determine whether subdural anesthesia wears off over a longer period of time with compared to epidural anesthesia.
Deep sedation of a patient in prone position without a secure airway is another challenge to the anesthesiologist. Main key technical points and the first priority of deep sedation on prone patient are the complete and adequate coverage of epidural anesthetic from the sacrum to the T12-L1 epidural space that would definitely cover the surgical field. If the epidural anesthesia was insufficient, any heavy sedatives do not work enough in reaching deep sedation and the patient certainly wake up and move which surely interrupt the operation proceeding. Furthermore, we utilized dexmedetomidine, which has little effect on respiratory function. However, only utilizing dexmedetomidine was not enough in reaching deep sedation and therefore we also used short-active midazolam and remifentanil. In addition, respiratory status was monitored by placing a capnogram close to the patient's mouth or nose in a semi-constrained environment, such as that created by the face protection foam. This way of monitoring respiration is not as accurate as monitoring the capnogram via an endotracheal tube. However, it continued to be crucial in our experience as it allowed us to manage airway issues far before we had an pulse oximeter changes by decreasing sedation, reopening a patient's airway, or replace a nasal cannula with a face mask for increased oxygenation. With the application of the capnogram to conscious sedation, we had no cases of conversion to general anesthesia due to airway issues.

Our experience shows that epidural anesthesia with half diluted local anesthetics results in satisfactory analgesia with minimal lower extremity motor blockade. An interesting point is that patients had a significant decrease in NRS immediately after surgery, an effect that also held true across age groups and ASA classes. This score includes incisional pain. This immediate improvement could be due to the blockade of nociception prior to incision, which has been reported to help with postoperative pain in the literature. ${ }^{20-23}$ Even though we do not compare this directly to patients undergoing general anesthesia, from our experience we know that patients continue to be in significant incisional pain immediately postoperatively, in which pain relief occurs over the first few days after surgery. The results of our study show that epidural anesthesia likely is beneficial in postoperative pain when compared to more conventional methods. Surprisingly, this includes patients undergoing fusions, with the significant muscle dissection and manipulation needed there, and even to endoscopic epidural procedures, where constant flow of irrigation threatens to wash away all anesthetic from the epidural space. ${ }^{24-27}$ 
In cases of revision surgery where there are significant epidural adhesions inhibiting the spread of anesthetic adequately, adding new analgesic method such as thoracolumbar interfascial plane block (TLIP), modified TLIP and Erector spinae plane block (ESP) ${ }^{28-30}$ together with epidural anesthesia will give us more complete and satisfied results of anesthesia and analgesia.

One criticism of epidural anesthesia is that it clouded a clinician's postoperative exam, which is crucial to rule out a postoperative hematoma or intraoperative injury. In our experience, utilizing half diluted anesthetics and ensuring that they are infused in the epidural and not the subarachnoid space utilizing fluoroscopy allowed for reasonable recovery times that did not affect the ability to perform a robust neurologic exam. Even in patients aged 70 or older, the slightly longer recovery time was not detrimental to the obtaining of a neurologic examination. We estimate that at least 7 milliliters of solution is needed full coverage from T12-L1. This is important for practitioners new to this technique to know as we demonstrated that decreasing the volume of epidural anesthesia is associated with decreased time to recovery. As such, a clinician should aim to decrease the amount of anesthetic used to obtain full coverage as much as possible. A limitation of our definition of recovery time is that it also includes the time for the patient to become alert and oriented, which is more the effect of the conscious sedation medications wearing off. As such, we believe that the time to motor recovery is even smaller than what is presented in our results.

\section{Conclusion}

In this retrospective study, we show that fluoroscopic guide epidural anesthesia in conjunction with conscious sedation is a safe and feasible approach to treating patients of all ages and an ASA class III or less undergoing lumbar spinal surgery less than $4 \mathrm{hrs}$ in procedural length and this includes even to the patients who have severe epidural adhesions such as in the case of revision surgery as well. Further larger randomized clinical trials are needed to study these effects further and compare this approach to the use of traditional general anesthesia.

\section{Disclosure}

The authors declare that they have no conflicts of interest regarding this work.

\section{References}

1. Attari MA, Mirhosseini SA, Honarmand A, et al. Spinal anesthesia versus general anesthesia for elective lumbar spine surgery: a randomized clinical trial. J Res Med Sci. 2011;16(4):524-529.
2. Horlocker TT, Cucchiara RF, Ebersold MJ. Vertebral column and spinal cord surgery. In: Cucciara F, Michenfelder JD, editors. Clinical Neuroanesthesia. New York: Churchill Livingstone; 1990:325-350.

3. Gulur P, Nishimori M, Ballantyne JC. Regional anaesthesia versus general anaesthesia, morbidity and mortality. Best Pract Res Clin Anaesthesiol. 2006;20:249-263. doi:10.1016/j.bpa.2005.10.002

4. Sztark F, Goff ML, Andre D, et al. Exposure to general anaesthesia could increase the risk of dementia in elderly: 18AP1-4. Eur J Anaesthesiol. 2013;30:245. doi:10.1097/00003643-201306001-00768

5. Mandel RJ, Brown MD, McCullough NC 3rd, et al. Hypotensive anesthesia and auto transfusion in spinal surgery. Clin Orthop Relat Res. 1981;154:27-33.

6. Scott NB, Kehlet H. Regional anaesthesia and surgical morbidity. $\mathrm{Br}$ J Surg. 1988;75:299-304.

7. Jellish WS, Thalji Z, Stevenson K, et al. A prospective randomized study comparing short- and intermediate-term perioperative outcome variables after spinal or general anesthesia for lumbar disk and laminectomy surgery. Anesth Analg. 1996;83:559-564. doi:10.1213/ 00000539-199609000-00021

8. Stauffer JL, Olson DE, Petty TL. Complications and consequences of endotracheal intubation and tracheostomy. Am J Med. 1981;70:65-76. doi:10.1016/0002-9343(81)90413-7

9. Flemming DC. Hazards of tracheal intubation. In: Orkin FK, Cooperman LH, editors. Complications in Anaesthesiology. Philadelphia: JB Lippincott Co.; 1983:165-172.

10. Lockhart PB, Feldbau EV, Gabel RA, et al. Dental complications during and after tracheal intubation. $J$ Am Dental Assoc. 1986;112:480-483. doi:10.14219/jada.archive.1986.0035

11. Johnson BA, Schellhas KP, Pollei SR. Epidurography and therapeutic epidural injections: technical considerations and experience with 5334 cases. Am J Neuroradiol. 1999;20(4):697-705.

12. Bromage PR. Epidural Analgesia. Philadelphia: WB Saunders; 1978:746.

13. Purnell RJ. Scoliosis correction and epidural analgesia. Prolonged block following Harrington rod insertion. Anesthesia. 1982;37:1115-1117. doi:10.1111/j.1365-2044.1982.tb01758.x

14. Johnson BA. Image-guided epidural injections. Neuroimaging Clin N Am. 2000;10(3):479-491.

15. Saberski LR, Kondamuri S, Osinubi OY. Identification of the epidural space: is loss of resistance to air a safe technique?: a review of the complications related to the use of air. Reg Anesth Pain Med. 1997;22:3-15. doi:10.1016/S1098-7339(06)80050-7

16. Masopust V, Hackel M, Netuka D, et al. Postoperative epidural fibrosis. Clin J Pain. 2009;25(7):600-606. doi:10.1097/AJP.0b013e3181a5b665

17. Manchikanti L, Bakhit CE. Percutaneous lysis of epidural adhesions. Pain Physician. 2000;3(1):46-64.

18. Lee F, Jamison DE, Hurley RW, et al. Epidural lysis of adhesions. Korean J Pain. 2014;27(1):3-15. doi:10.3344/kjp.2014.27.1.3

19. Milants WP, Parizel PM, de Moor J, et al. Epidural and subdural contrast in myelography and CT myelography. Eur $J$ Radiol. 1993;16:147-150. doi:10.1016/0720-048X(93)90013-D

20. Abram SE, Yaksh TL. Morphine, but not inhalational anesthesia, blocks post-injury facilitation. Anesthesiology. 1993;78(4):713-721. doi:10.1097/00000542-199304000-00015

21. Kakiuchi M, Abe K. Pre-incisional caudal epidural blockade and the relief of pain after lumbar spine operations. Int Orthop. 1997;21:62-66. doi:10.1007/s002640050121

22. Kundra P, Gurnani A, Bhattacharya A. Preemptive epidural morphine for postoperative pain relief after lumbar laminectomy. Anesth Analg. 1997;85:135-138. doi:10.1097/00000539-199707000-00024

23. Richard CE, Bromley LM, Woolf CJ. Preoperative morphine pre-empts postoperative pain. Lancet. 1993;342:73-75. doi:10.1016/ 0140-6736(93)91284-S

24. Chan VWS, Nazarnia S, Kaszas Z, et al. The impact of saline flush of the epidural catheter on resolution of epidural anesthesia in volunteers: a dose-response study. Anesth Analg. 1999;89:1006-1010. doi:10.1097/00000539-199910000-00034 
25. Johnson MD, Burger GA, Mushlin PS, et al. Reversal of bupivacaine epidural anesthesia by intermittent epidural injections of crystalloid solutions. Anesth Analg. 1990;70:395-399. doi:10.1213/00000539199004000-00009

26. Sitzman TB, DiFazio CA, Playfair PA, et al. Reversal of lidocaine with epinephrine epidural anesthesia using epidural saline washout. Reg Anesth Pain Med. 2001;26:246-251. doi:10.1097/00115550-200105000-00008

27. Rodríguez J, Rodríguez V, Naveira A, et al. Epidural washout with high volumes of saline to accelerate recovery from epidural anaesthesia. Acta Anaesth Scand. 2001;45:893-898. doi:10.1034/ j.1399-6576.2001.045007893.x
28. Hand WR, Taylor JM, Harvey NR, et al. Thoracolumbar interfascial plane (TLIP) block: a pilot study in volunteers. Can J Anesth. 2015;62:1196-1200. doi:10.1007/s12630-015-0431-y

29. Li C, Jia J, Qin Z, et al. The use of ultrasound-guided modified thoracolumbar interfascial plane block for multi-level lumbar spinal surgery. C Clin Anesth. 2018;46:49-51. doi:10.1016/j. jclinane.2018.01.018

30. Cesur S, Yayik AM, Ozturk F, et al. Ultrasound-guided low thoracic erector spinae plane block for effective postoperative analgesia after lumbar surgery: report of five cases. Cureus. 2018;10(11):e3603.

\section{Publish your work in this journal}

The Journal of Pain Research is an international, peer reviewed, open access, online journal that welcomes laboratory and clinical findings in the fields of pain research and the prevention and management of pain. Original research, reviews, symposium reports, hypothesis formation and commentaries are all considered for publication. The manuscript

Submit your manuscript here: https://www.dovepress.com/journal-of-pain-research-journa management system is completely online and includes a very quick and fair peer-review system, which is all easy to use. Visit http:// www.dovepress.com/testimonials.php to read real quotes from published authors. 